\title{
Les techniques de génétique moléculaire au service de l'épidémiologie des trypanosomoses. Intérêt de l'étude du polymorphisme des microsatellites des glossines
}

\author{
P. Solano ${ }^{1,2^{*}}$ G. Cuny ${ }^{3}$ G. Duvallet ${ }^{2}$ \\ D. Cuisance ${ }^{2}$ S. Ravel ${ }^{3}$ I. Sidibé ${ }^{1}$ S.M. Touré ${ }^{1}$
}

Mots-clés

Trypanosomose - Glossina palpalis gambiensis - PCR - Microsatellite Variation génétique - Epidémiologie.

\section{Résumé}

L'introduction des techniques moléculaires a apporté de nouvelles connaissances sur l'épidémiologie des trypanosomoses. Dans le domaine du diagnostic, la technique d'amplification en chaîne par polymérase (ou polymerase chain reaction) apporte une sensibilité supérieure à celle des techniques classiques, et permet aussi de différencier des trypanosomes morphologiquement identiques ayant des importances économiques très différentes. Chez les vecteurs cycliques, les glossines, on soupçonne l'existence de populations plus dangereuses que d'autres au sein d'une même espèce ; les auteurs décrivent et proposent d'appliquer la technique du polymorphisme des marqueurs microsatellites à l'évaluation des conséquences de la variabilité intraspécifique des mouches tsé-tsé sur l'épidémiologie de la trypanosomose.

\section{INTRODUCTION}

Les trypanosomoses africaines sont causées par des protozoaires flagellés du genre Trypanosoma et sont transmises principalement par des insectes diptères, les glossines. Leur importance économique en Afrique subsaharienne est considérable puisqu'elles provoquent chez l'homme la maladie du sommeil (50 millions de personnes exposées) et chez les animaux domestiques le nagana ou trypanosomose animale ; certains experts estiment les pertes économiques du secteur de l'élevage dues aux trypanosomoses à 1 milliard de dollars par an à l'échelle du continent (3).

Il est donc nécessaire d'être capable de diagnostiquer la maladie lorsqu'elle existe, mais aussi et surtout de pouvoir la contrôler en abaissant son importance à un niveau n'empêchant pas la production. Un moyen pour y parvenir est de lutter contre les cibles définies que sont certaines populations de glossines propageant l'épizootie.

1. CIRDES, 01 BP 454, Bobo-Dioulasso 01, Burkina Faso

2. CIRAD-EMVT, Campus international de Baillarguet, BP 5035, 34032 Montpellier Cedex 1, France

3. ORSTOM Montpellier, BP 5045, 34032 Montpellier Cedex, France

* Auteur assurant la correspondance : voir adresse 1

Tél. : 226972287 ; fax: 226972320

\section{- UTILISATION DE LA BIOLOGIE MOLECULAIRE DANS LA DETECTION ET L'IDENTIFICATION DU PARASITE}

Ces dernières années, le développement des techniques d'étude moléculaire, notamment la PCR (polymerase chain reaction) (16, 21) ou ACP (amplification en chaîne par polymérase), a généré des connaissances nouvelles dans l'épidémiologie des trypanosomoses. Il est maintenant possible de distinguer chez les glossines des trypanosomes morphologiquement similaires mais différents par leurs caractères nosologiques et leur spectre d'hôtes : il en est ainsi, dans le sous-genre Nannomonas, de l'espèce $T$. simiae, pathogène pour les Suidés domestiques mais inoffensive pour les Ruminants (8), et de l'espèce $T$. congolense qui infecte également les Suidés, mais qui compte parmi les trypanosomes les plus pathogènes pour le cheptel bovin. Dans le domaine du diagnostic, la PCR permet même de détecter différents sous-groupes taxonomiques de $T$. congolense $(11,12,13)$ : en Afrique de l'Ouest sont présents principalement le groupe «Savane » et le groupe « Forêt» $(14,17,19,22,23)$; on les soupçonne également d'avoir des pathogénicités différentes (18) et des études sont actuellement en cours pour confirmer ces présomptions.

De nombreuses applications de la technique PCR ont été développées. Cette technique prometteuse dans le domaine du diagnostic par sa sensibilité et sa spécificité reste pour l'instant limitée dans 
ses applications par son coût relativement élevé. Pour le diagnostic des trypanosomes, les séquences d'ADN satellite (courte séquence répétée en tandem un grand nombre de fois) ont été utilisées. Ces séquences sont spécifiques du groupe de trypanosomes recherchés et leur utilisation comme cible d'amplification, à l'aide d'amorces connues, permet d'augmenter la sensibilité du test. Ces informations (diagnostic fiable et rapide, identification des agents pathogènes) permettent d'apprécier rapidement la situation épidémiologique et constituent des outils stratégiques d'aide à la décision.

Les RAPD (random amplified polymorphic DNA) $(31,32)$ sont également dérivés de la PCR. La réaction fait intervenir une seule amorce, courte et de séquence aléatoire. Du fait de leur faible longueur, ces amorces ont une probabilité importante de trouver plusieurs séquences homologues dans le génome. On obtient ainsi des profils à bandes multiples, spécifiques de souches ou d'ensembles de souches de trypanosomes. Cette technique a notamment permis de montrer que $T$. congolense forme une espèce polyphylétique : on ne trouve pas d'ancêtre unique, commun aux lignées actuelles, mais plusieurs. Ceci a pour conséquence la très faible probabilité de trouver une séquence diagnostique de l'espèce $T$. congolense dans son ensemble (22).

\section{- UNE APPRO CHE CO MPLEMENTAIRE : LA VARIABILITE GENETIQUE DES VECTEU RS}

Si on est capable d'identifier au niveau intraspécifique chez les trypanosomes certains taxons dont l'épidémiologie ou la pathogénie sont différentes, il n'en est pas encore de même chez les vecteurs majeurs que sont les glossines. Or pour un taxon donné d'insectes, le déterminisme génétique de la capacité à s'infecter et à transmettre les trypanosomes a déjà fait l'objet de plusieurs observations. Janssen et Wijers trouvent des différences de transmission d'un même clone de T. simiae entre Glossina brevipalpis et $G$. pallidipes et suggèrent que l'espèce de glossine peut être un facteur conditionnant la pathogénicité de la souche transmise (9). Avec le même parasite, Moloo et coll. observent une différence de susceptibilité entre deux populations allopatriques de G. pallidipes (15). Ces deux populations montrent aussi des différences de transmission d'un clone de $T$. congolense. Reifenberg et coll., en comparant la capacité vectorielle de plusieurs taxons de glossines vis-à-vis de $T$. congolense montrent, entre autres, que les deux sous-espèces $G$. morsitans submorsitans et $G$. m. morsitans n'ont pas la même capacité à transmettre le parasite au laboratoire (17). En allant plus loin, au niveau infra-taxon, Elsen et coll. observent des différences significatives de réceptivité du même clone de T. brucei gambiense chez deux populations d'insectarium de G. palpalis gambiensis provenant du même site géographique mais ayant été maintenues séparément plusieurs années (5). Ces auteurs, en utilisant les isoenzymes et l'aspect des chromosomes, trouvent certaines différences génétiques qu'ils attribuent essentiellement à un phénomène de dérive, classique dans des populations élevées au laboratoire. Makumyaviri et coll. comparent une population sauvage de G. m. morsitans à un phénotype mutant salmon (couleur de l'œil), et observent une meilleure colonisation de l'intestin moyen par T. brucei chez le phénotype mutant, qui serait liée à une modification du métabolisme du tryptophane (10).

Il semble donc intéressant d'appréhender la variabilité génétique des populations de vecteurs, avec pour objectif dans un taxon donné :
1. De clarifier les relations fines parasite-vecteur (compétence vectorielle), ce qui pourrait permettre une meilleure définition des cibles des campagnes de contrôle de la trypanosomose. La compétence vectorielle est l'aptitude physiologique d'un vecteur à acquérir le pathogène et à le transmettre (20).

2. D'essayer de distinguer des populations de valeur épidémiologique différente (capacité vectorielle). La capacité vectorielle est le nombre de nouvelles infections par situation et par jour du fait d'un vecteur ; elle dépend de la compétence vectorielle et de facteurs du milieu (20). Le moyen est d'identifier au sein d'un taxon donné une structuration génétique des populations en analysant des fréquences alléliques. Le fait de trouver des fréquences alléliques significativement différentes pour un ensemble d'individus dans un espace donné peut montrer que la reproduction ne se fait pas de manière aléatoire entre ces individus ; il y a donc des sousgroupes différents au sein de la même espèce (ou sous-espèce) dans le même espace ou sur des espaces voisins qui peuvent indiquer :

- des préférences différentes d'hôte nourricier : risque épidémiologique ;

- des préférences différentes de biotopes : distribution spatiale et temporelle ;

- un comportement d'évitement de leurres artificiels (pièges, écrans, etc.), ou vivants (bétail imprégné d'insecticides) : résistance aux mesures de lutte ;

- une différence d'aptitude à la maturation des trypanosomes : compétence vectorielle.

\section{- LA TECHNIQUE}

Une séquence microsatellite est constituée de courtes répétitions $(<6)$ de 2,3 , ou 4 nucléotides $(28,29)$. Ces motifs sont localisés à différents endroits du génome, constituant chacun un locus. Ces séquences existent chez tous les eucaryotes où on les a recherchées, et se sont révélées de très bons marqueurs polymorphes pour des études de génétique des populations. Les microsatellites présentent, pour un locus donné, un polymorphisme de longueur dû à la variation du nombre de répétitions, permettant ainsi de définir des allèles.

Exemples de séquences microsatellites :

Motif dinucléotidique

AT AT AT AT AT AT AT AT AT AT

Motif trinucléotidique

CAG CAG CAG CAG CAG CAG CAG CAG CAG

Après avoir détecté, isolé et séquencé ces motifs microsatellites, des amorces (ou primers) sont définies de part et d'autre du motif répété (figure 1), mais pas dans son voisinage immédiat (instabilité des séquences flanquant le microsatellite). Une amplification par PCR est réalisée et on obtient sur gel une bande ou deux dont les tailles sont directement proportionnelles au nombre de répétitions du motif. Ces signaux d'amplification (ou allèles) vont donc varier entre les individus, en fonction du nombre de répétitions que ceux-ci possèdent.

Par exemple, un individu (AT)10 donne une bande de 180 paires de bases : cette taille correspond à un motif microsatellite qui est ici de 20 nucléotides ( 2 x 10), plus les amorces et la séquence flanquante totalisant dans cet exemple 160 nucléotides (taille invariante entre les individus). 


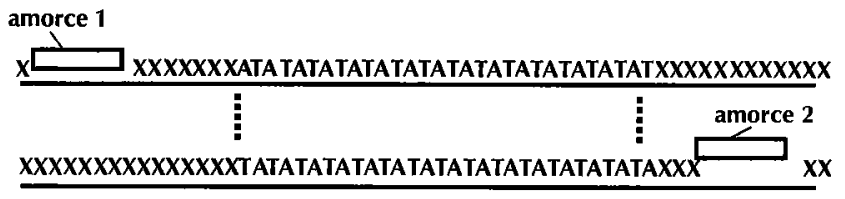

Figure 1 : définition des amorces $P C R$ autour d'un motif microsatellite (AT).

Un autre individu (AT)14 donnera un signal de 188 paires de bases $(=160+(2 \times 14))$; leur motif de répétition diffère de 4 unités. Cette différence se traduit par une différence de migration visible sur gel. Dans ces deux cas, les individus ne présentent qu'une seule bande et sont alors considérés comme homozygotes pour ce locus.

\section{a AVANTAGES POUR LES ETUDES DE GENETIQUE DES POPULATIONS}

Les marqueurs microsatellites peuvent être utilisés en génétique des populations car ils présentent les caractéristiques suivantes :

- ils sont supposés être sélectivement neutres, car très abondants et situés dans des régions non-codantes, sauf quelques exceptions de répétitions associées à des maladies (dystrophie myotonique, cancers) $(6,28)$. La sélection n'influe donc théoriquement pas sur les fréquences alléliques ;

- les allèles sont transmis d'une génération à l'autre suivant les lois mendéliennes classiques. Cette caractéristique a été confirmée sur une population de G. palpalis gambiensis maintenue en insectarium. Ce sont des marqueurs codominants, ce qui permet de distinguer des homozygotes ( 1 bande) et des hétérozygotes ( 2 bandes) (figure 2).

Prenons par exemple un père $180 \mathrm{pb}$ (motif : (AT) 10 ) et une mère $188 \mathrm{pb}$ (AT)14. L'enfant sera 180-188 (10-14). L'enfant chez un taxon diploïde hérite d'un chromosome paternel et d'un maternel. Dans ce cas, il présente donc deux bandes.

Les séquences microsatellites présentent une très forte variabilité et un haut degré de polymorphisme. Par exemple, dans les populations naturelles de Glossina palpalis gambiensis, il y en a général un maximum de 4 à 5 allèles par locus isoenzymatique (7), et il y en a entre 10 et 40 par locus microsatellite.

L'analyse PCR permet de travailler sur de très petites quantités de matériel (1 patte pour les glossines) et permet de se passer d'une chaîne de froid pour les études de terrain. Ceci ramène le coût des analyses à des valeurs raisonnables. A titre d'exemple, le prix de revient d'une étude portant sur 100 individus avec 3 loci microsatellites est d'environ $2500 \mathrm{FF}$ (environ 430 dollars LS) en consommables ; ce prix est calculé pour un laboratoire équipé pour la biologie moléculaire, et possédant déjà les amorces PCR nécessaires.

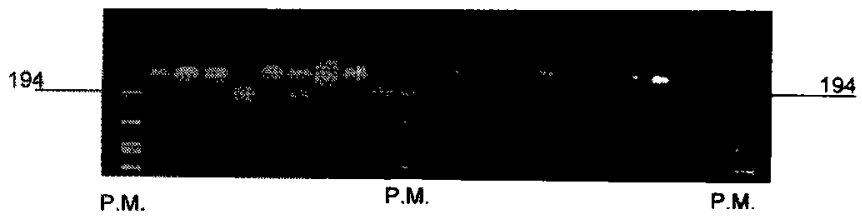

Figure 2 : révélation sur gel d'agarose des allèles microsatellites amplifiés par PCR ; 194 = taille des bandes à ce niveau indiqué par le marqueur de poids moléculaire (P.M.). On distingue des homozygotes ( 1 bande) et des hétérozygotes ( 2 bandes).
Les séquences microsatellites trouvées dans un taxon donné peuvent être conservées dans d'autres taxons phylogénétiquement proches, ainsi que leurs séquences flanquantes ; ceci permet d'étendre ce type d'étude aux espèces voisines, en évitant les étapes d'isolement, clonage et séquençage des microsatellites. Ainsi, trois couples d'amorces définis chez $G$. palpalis gambiensis permettent d'obtenir des produits d'amplification également chez G. p. palpalis et G.f. fuscipes (24).

\section{AUTRES APPLICATIONS}

L'identification de plusieurs séquences microsatellites, donc plusieurs loci, conduit à la connaissance du génotype (la composition allélique) des individus à ces différents loci. Ce marquage génétique de population peut être appliqué notamment pour :

- identifier des populations de réinvasion après une campagne de lutte (4) ;

- modéliser les flux de gènes à l'intérieur d'une population ou entre des populations afin de prévoir l'effet de certaines méthodes de contrôle, par exemple lorsqu' on veut introduire des éléments (transposons) dans les populations de vecteurs rendant ceux-ci réfractaires aux parasites $(1,26)$. Dans ce cas, il faut savoir si ce gène va se transmettre facilement à tous les individus d'une population et à quelle vitesse, ou si des barrières existent qui vont empêcher son passage. Il a été envisagé chez les moustiques d'introduire des gènes de zoophilie (Anopheles quadriannulatus) dans des populations " anthropophiles" (An. gambiae s.s.) (2). Cela suppose donc que 1'on reconnaisse ces populations génétiquement. Pourquoi ne pas imaginer que l'on puisse obliger les glossines à se nourrir exclusivement sur reptiles !

- enfin, cette approche présente un intérêt pour les équipes travaillant sur les cartes génomiques des mammifères, bien sûr (30), mais aussi des vecteurs. Il existe ainsi une carte détaillée du chromosome X chez An. gambiae grâce aux microsatellites (33).

\section{CONCLUSION}

Le développement des techniques moléculaires a ouvert de nouveaux horizons pour la compréhension de l'épidémiologie des trypanosomoses animales ; les informations apportées par ces technologies deviennent indispensables et constituent des outils d'aide en stratégie d'intervention. Toutefois, les variations intraspécifiques existant chez les glossines, vectrices des trypanosomoses, ne sont pas encore bien connues, et pourraient avoir une influence déterminante sur leur capacité à disséminer la maladie. Afin d'évaluer les conséquences épidémiologiques de ces variations intraspécifiques, les auteurs proposent d'appliquer aux glossines le polymorphisme des marqueurs microsatellites, cette technique ouvrant des perspectives prometteuses par les informations potentielles qu'elle offre.

\section{Remerciements}

Ce travail a bénéficié d'un financement du CIRAD (Action thématique programmée), du CNRS (programme interdisciplinaire : Environnement, vie et sociétés), et de l'AUPELF-UREF (Laboratoire associé francophone $\mathrm{n}^{\circ} 306$ ) pour lesquels les auteurs expriment leurs plus vifs remerciements. Sont également remerciés pour leurs conseils sur ce manuscrit, Dr J.L. Frézil (ORSTOM) et Dr T. Lefrançois (Ecole vétérinaire de Maisons-Alfort). 


\section{BIBLIO GRAPHIE}

1. COLLINS F.H., 1994. Prospects for malaria control through the genetic manipulation of its vectors. Parasitol. today, 10: 370-371.

2. CURTIS C.F., 1994. The case for malaria control by genetic manipulation of its vectors. Parasitol. today, 10: 371-374.

3. DE HAAN C., BEKURE S., 1991. Animal health services in SubSaharan Africa: Initial experiences with new approaches. Washington DC, USA, World Bank.

4. DUMAS V., HERDER S., BEBBA A., CADOUX-BARNABE C. BELLEC C., CUNY G., 1997. Polymorphic microsatellites in Simulium damnosum s.l. and their use for differenciating two savannah populations. Implications for epidemiological studies. Genome (sous presse)

5. ELSEN P., ROELANTS P., DE LIL E., DUJARDIN J.P., LE RAY D. CLAES Y., 1994. Cytogenetic and isozymic comparisons of two laboratory lines of Glossina palpalis gambiensis. Ann. trop. Med. Parasitol., 88: 511-522.

6. EPPLEN J.E., 1993. Diagnostic applications of repetitive DNA sequences. Clin. Chem. Acta, 209: S5-S13.

7. GOODING R.H., 1981. Genetic polymorphism in three species of tsetse flies (Diptera: Glossinidae) in Upper Volta. Acta trop., 38 149-161.

8. ITARD J., 1981. Trypanosomoses animales africaines. In : Précis de parasitologie vétérinaire tropicale, tome II. Paris, France, Ministère de la coopération et du développement, p. 305-468.

9. JANSSEN J.A.H.A., WIJERS D.J.B., 1974. Trypanosoma simiae at the Kenya coast. A correlation between virulence and the transmitting species of Glossina. Ann. trop. Med. Parasitol., 68: 5-19.

10. MAKU M YAVIRI A.M., DISTELM ANS W., CLAES Y., D'HAESELEER F., LE RAY D., GOODING R.H., 1984. Capacité vectorielle du type sauvage et du mutant salmon de Glossina morsitans morsitans Westwood, 1850 dans la transmission de Trypanosoma brucei Plimmer et Bradford, 1899. Cah. ORSTOM, sér. Ent. méd. Parasitol., 22 283-288

11. MAJIWA P.A.O., MAINA M., WAITUMBI J.N., MIHOK S., ZWEYGARTH E., 1993. Trypanosoma (Nannomonas) congolense: molecular characterization of a new genotype from Tsavo, Kenya. Parasitology, 106: 151-162.

12. MASIGA D.K., SMYTH A.J., HAYES P., BROMIDGE T.J. GIBSON W.C., 1992. Sensitive detection of trypanosomes in tsetse flies by DNA amplification. Int. J. Parasitol., 22: 909-918.

13. MCNAMARA J.J., MOHAMMED G., GIBSON W.C., 1994 Trypanosoma (Nannomonas) godfreyi sp. nov. from tsetse flies in The Gambia: biological and biochemical characterisation. Parasitology, 109: 497-509.

14. MCNAMARA J.J., SNOW W.F., 1991. Improved identification of Nannomonas infections in tsetse flies from the Gambia. Acta trop., 48: 127-136.

15. MOLOO S.K., ZWEYGARTH E., SABWA C.L., 1995. A comparison of susceptibility to stocks of Trypanosoma simiae of Glossina pallidipes originating from allopatric populations in Kenya. Med. vet. Entomol., 9: 224-228.

16. MULLIS L.B., FALO ONA F.A., 1987. Specific synthesis of DNA in vitro via a polymerase catalyzed chain reaction. Methods Enzymol. 155: 335-350.
17. REIFENBERG J.M., CUISANCE D., FREZIL J.L., CUNY G., DUVALLET G., 1997. Comparison of the susceptibility of different Glossina species to simple and mixed infections with Trypanosoma (Nannomonas) congolense Savannah and riverine-forest types. Med. vet. Entomol., 11: 246-252.

18. REIFENBERG J.M., SO LANO P., BAUER B., KABORE I., CUNY G., DUVALLET G., CUISANCE D., 1997. Apport de la technique PCR pour une meilleure compréhension de l'épizootiologie des trypanosomoses bovines : exemple de la zone d'aménagement pastoral de Yalé au Burkina Faso. Revue Elev. M éd. vet. Pays trop., 50 : 14-22.

19. REIFENBERG J.M., SOLANO P., DUVALLET G., CUISANCE D., SIMPORE J., CUNY G., 1997. Molecular characterization of trypanosome isolates from naturally infected domestic animals in Burkina Faso. Vet. Parasitol., 71: 251-262.

20. REISEN W.K., 1989. Estimation of vectorial capacity : introduction. Bull. Soc. Vector Ecol., 14: 39-40.

21. SAIKI R.K., GELFLAND D.H., STOFFEL S., 1988. Primer-directed enzymatic amplification of DNA with a thermostable DNA polymerase. Science, 239: 487-491.

22. SIDIBE I., 1996. Variabilité génétique de Trypanosoma congolense, agent de la trypanosomose animale : implications taxonomiques et épidémiologiques. Thèse doct., Université de Montpellier II, France, $92 \mathrm{p}$

23. SO LANO P., ARGIRO L., REIFEN BERG J.M., YAO Y., DUVALLET G., 1995. Field application of the polymerase chain reaction (PCR) to the detection and characterization of trypanosomes in Glossina longipalpis in Côte d'Ivoire. Mol. Ecol., 4: 781-785.

24. SO LANO P., DUVALLET G., DUMAS V., CUISANCE D., CUNY G., 1997. Microsatellite markers for genetic population studies in Glossina palpalis (Diptera: Glossinidae). Acta trop., 65: 175-180.

25. SO LAN O P., REIFEN BERG J.M., AM SLER-D ELAFO SSE S., KABO RE I., CUISANCE D., DUVALLET G., 1996. Trypanosome characterization by polymerase chain reaction in Glossina palpalis gambiensis and G. tachinoides from Burkina Faso. Med. vet. Entomol., 10: 354-358.

26. SPIELMAN A., 1994. Why entomological antimalaria research should not focus on transgenic mosquitoes. Parasitol. today, 10: 374-376.

27. TAUTZ D., 1989. Hypervariability of simple sequences as a general source for polymorphic DNA markers. Nucl. Acids Res., 17: 6463-6471.

28. WATKINS W.S., BAMSHAD M., JORDE L.B., 1995. Population genetics of trinucleotide repeat polymorphisms. Hum. mol. Gen., 4: 1485-1491.

29. WEBER J.L., 1990. Informativeness of human (dC-dA)n-(dG-dT)n polymorphisms. Genomics, 7: 524-530.

30. WEISSENBACH J., DE CHENAY A., 1992. Le génome humain balisé par des microsatellites. La Recherche, 261 : 84-85.

31. WELSH J., MCCLELLAND M., 1990. Fingerprinting genomes using PCR with arbitrary primers. Nucl. Acids Res., 18: 7213-7218.

32. WILLIAMS J.G.K., KUBELIK A.R., LIVAK K.J., RAFALSKI J.A., TINGEY S.V., 1990. DNA polymorphisms amplified by arbitrary primers are useful genetic markers. Nucl. Acids Res., 18: 6531-6535.

33. ZHENG L., COLLINS F.H., KUMAR V., KAFATOS F.C., 1993. A detailed genetic map for the $X$ chromosome of the malaria vector, Anopheles gambiae. Science, 261: 605-608.

Reçu le 12.1.98, accepté le 24.2.98 


\section{Summary}

Solano P., Cuny G., D uvallet G., Cuisance D., Ravel S., Sidibé I., Touré S.M. M olecular genetic techniques applied to the epidemiology of trypanosomoses. Advantages of the microsatellite polymorphism study in tsetse flies

A further understanding of trypanosomosis epidemiology has been brought about by the development of molecular techniques. With regard to diagnosis, the polymerase chain reaction (PCR) combines the advantages of greater sensibility than classical techniques and the possibility to differentiate trypanosomes with similar morphology but a very different economic impact. Populations of cyclic vectors (tsetse flies) have been suspected to exist with varying degrees of dangerousness within the same species. The authors describe and propose to apply the technique of microsatellite DNA polymorphism to assess the consequences of tsetse intraspecific variability on the epidemiology of trypanosomosis.

Key words: Trypanosomosis - Glossina palpalis gambiensis PCR - M icrosatellite - Genetic variation - Epidemiology.

\section{Resumen}

Solano P., Cuny G., Duvallet G., Cuisance D., Ravel S., Sidibé I., Touré S.M. Las técnicas de genética molecular al servicio de la epidemiología de las tripanosomosis. Importancia del polimorfismo de los microsatélites de las glosinas

Las técnicas moleculares han aportado nuevos conocimientos sobre la epidemiología de las tripanosomosis. En el campo del diagnóstico, la técnica de amplificación en cadena por polimerasas (o reacción en cadena de las polimerasas) aporta una sensibilidad superior que las técnicas clásicas, permitiendo también la diferenciación de los tripanosomas morfológicamente idénticos, con diferentes importancias económicas. En el caso de los vectores cíclicos, las glosinas, se sospecha la existencia de algunas poblaciones más peligrosas que otras, en el seno de una mima especie. Los autores describen y proponen la aplicación de la técnica del polimorfismo de los microsatélites marcadores, para la evaluación de las consecuencias de la variabilidad intra específica de las tse-tse sobre la epidemiología de la tripanosomosis.

Palabras clave: Tripanosomosis - Glossina palpalis gambiensis PCR - Microsatélite - Variación genética - Epidemiología. 